\title{
Hochselektiver Festelektrolytsensor für die Analyse von Gasgemischen
}

\author{
Matthias Schelter ${ }^{1}$, Jens Zosel', Wolfram Oelßner ${ }^{1}$, Ulrich Guth ${ }^{1,2}$, Michael Mertig ${ }^{1,2}$ \\ ${ }^{1}$ Kurt-Schwabe-Institut für Mess- und Sensortechnik e.V. Meinsberg, \\ Kurt-Schwabe-Str. 4, 04736 Waldheim, Deutschland \\ ${ }^{2}$ Technische Universität Dresden, Professur für Physikalische Chemie, Mess- und Sensortechnik \\ Technische Universität Dresden, Physikalische Chemie, 01062 Dresden, Deutschland \\ schelter@ksi-meinsberg.de
}

\begin{abstract}
Zusammenfassung
An einem kommerziell verfügbaren Festelektrolytsensor wurden im Durchflussmodus systematische Untersuchungen mittels Cyclovoltammetrie an $\mathrm{H}_{2}$ - und $\mathrm{O}_{2}$-haltigen Gasgemischen durchgeführt. Dabei wurden die Einflussgrößen Zelltemperatur, Elektrodengröße und Potentialvorschubgeschwindigkeit variiert und Bedingungen gefunden, bei denen es im Cyclovoltammogramm zur charakteristischen Peakausbildung für die jeweiligen Komponenten kommt. Während sich die $\mathrm{H}_{2}$-Oxidationspeaks bei Potentialvorschubgeschwindigkeiten bis $100 \mathrm{mV} / \mathrm{s}$ ausbilden, treten $\mathrm{O}_{2}$-Reduktionspeaks im Bereich zwischen 200 und $1000 \mathrm{mV} / \mathrm{s}$ auf. Im unteren Konzentrationsbereich gibt es für jedes dieser Gase einen linearen Zusammenhang zwischen der Konzentration und den auswertbaren Peakeigenschaften Höhe und Fläche. Es konnte gezeigt werden, dass trotz der katalytisch hochaktiven Platinelektroden $\mathrm{H}_{2}$ bei unterschiedlichen Beimengungen von $\mathrm{O}_{2}$ im Messgas selektiv erfasst werden kann.
\end{abstract}

Keywords: Festelektrolytsensor, Selektivität, Querempfindlichkeit, YSZ, Cyclovoltammetrie

\section{Einleitung}

Bei der Regelung von mobilen und stationären Verbrennungsprozessen sowie in der Medizin, der Umweltmesstechnik, der Sicherheitstechnik, der chemischen Industrie, der Bioprozesstechnik und hier insbesondere auch in der Biogastechnik besteht häufig das Problem, redoxaktive Gase wie Wasserstoff und Sauerstoff, die nebeneinander in Gasgemischen vorliegen, gleichzeitig bestimmen zu müssen. Festelektrolytsensoren mit Platinelektroden, die mit stationären Messmethoden wie Potentiometrie, Amperometrie oder Coulometrie betrieben werden, sind jedoch nicht dazu geeignet, gleichzeitig mehrere Analyte mit hinreichender Selektivität zu bestimmen. Beispielsweise stellt sich an einer Zelle des Typs „Messgas,Pt|YSZ|Pt,Luft" eine Zellspannung entsprechend dem Gleichgewichts-Sauerstoffpartialdruck im Messgas ein. Dabei tragen alle im Messgas vorhandenen Spezies, die an der heißen Platinelektrode des Festelektrolytsensors mit Sauerstoff reagieren, zum Sauerstoffpartialdruck und folglich zur Potentialbildung bei. Aus der gemessenen Zellspannung kann demnach nicht auf Anzahl, Art und Konzentration der im Messgas enthaltenen Spezies geschlossen werden.
Im Gegensatz zu den oben genannten stationären Messmethoden besteht die Grundidee der hier vorgestellten Untersuchungen darin, durch Anwendung der Cyclovoltammetrie [1] die Selektivität von Festelektrolytsensoren zu erhöhen. Durch die periodische Variation der an den Sensor angelegten Zellspannung $U_{p}(t)$ wird unter Ausnutzung verschiedener Reaktionskinetiken und/oder unterschiedlicher Elektrodenpotentiale die zeitlich getrennte selektive Bestimmung mehrerer Analyte ermöglicht. Es wird gezeigt, dass mit dieser Methode Brenngase wie $\mathrm{H}_{2}$ auch bei Anwesenheit von $\mathrm{O}_{2}$ an katalytisch hochaktiven Elektroden bestimmt werden können.

\section{Experimentelles}

Die in den vorgestellten Untersuchungen verwendete, in Abb. 1 dargestellte Messanordnung enthält eine beheizbare rohrförmige Zelle (ZIROX - Sensoren \& Elektronik $\mathrm{GmbH}$, Greifswald, Deutschland) des Typs "Messgas, Pt| YSZ|Pt,Luft", durch die das Messgas mit Volumenströmen zwischen 5 und $50 \mathrm{~mL} / \mathrm{min}$ hindurchströmt [2]. Der Grundkörper des Festelektrolytsensors bestand aus einem oxidionenleitenden Material (Yttriumoxid-stabilisiertes Zirkoniumdioxid, YSZ), das auf eine definierte Temperatur zwischen 650 und $850{ }^{\circ} \mathrm{C}$ aufgeheizt wurde. Das elektrochemische 
Messsystem Reference 600 $00^{\text {TM }}$ (Gamry Instruments, Warminster, USA) diente zur Aufnahme der Cyclovoltammogramme sowie zur Aufzeichnung der Ruhespannungen der Zelle.

\section{Festelektrolytzelle}

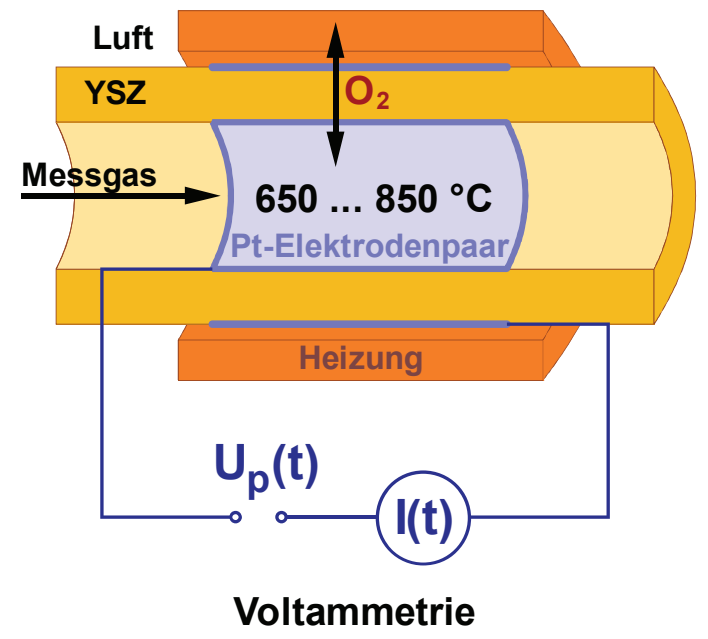

Abb. 1: Messanordnung des Festelektrolytsensors (Elektrodenflächen innen/außen: $7,5 \mathrm{~cm}^{2} / 11,3 \mathrm{~cm}^{2}$ ) mit cyclovoltammetrischer Messeinrichtung; $U_{p}(t)$ : periodisch variierte Zellspannung; I(t): Messsignal.

Die in dieser Arbeit verwendeten Gase $\mathrm{N}_{2}$, synthetische Luft und 1001 Vol.-ppm $\mathrm{H}_{2}$ in $\mathrm{N}_{2}$ (alle von Rießner-Gase $\mathrm{GmbH}$, Lichtenfels, Deutschland) wurden mittels Masseflussreglern (Brooks Instrument Company, Hatfield, USA) gemischt, woraus definierte Prüfgaskonzentrationen resultierten.

\section{Ergebnisse}

\section{I-U-Kennlinien des Festelektrolytsensors}

Während der Aufnahme eines Cyclovoltammogramms ändern sich infolge der Potentialveränderung die Reaktionsmechanismen der Elektrodenprozesse. Für das Verständnis der komplexen Entwicklung dieser Prozesse ist es zweckmäßig, zunächst die in Abb. 2 gezeigte Strom-Spannungs-Kennlinie der Zelle mit getrenntem Gasraum des Typs „Stickstoff,Pt| YSZ|Pt, Luft" zu betrachten.

Bei positiver Polarisation der Messgaselektrode wird $\mathrm{O}_{2}$ von der Referenzelektrode durch den Festelektrolyt in den Messgasstrom gepumpt. Dabei wird Sauerstoff an der Referenzelektrode zu Oxidionen reduziert, die bedingt durch die $\mathrm{O}^{2-}$-Leitfähigkeit des Festelektrolyts zur Messgaselektrode geleitet und dort $\mathrm{zu} \mathrm{O}_{2}$ oxidiert werden. Strombestimmend wirkt in diesem Spannungsbereich hauptsächlich der ohmsche Zellwiderstand, aus dem ein linear mit der Spannung ansteigender Stromfluss resultiert. Bei negativer Polarisation $\left(\mathrm{O}_{2}\right.$ wird aus dem Messgasstrom heraus und zur Referenzelektrode gepumpt) kann bei Abwesenheit von $\mathrm{O}_{2}$ im Messgas kein lonenstrom durch den Festelektrolyt fließen (blockierende Elektrode). Eine linear ansteigende Überspannung in die anodische Richtung führt gemäß der Butler-Volmer-Gleichung zu einem exponentiell ansteigenden Stromfluss. Oberhalb einer Spannung von etwa $0 \mathrm{~V}$ folgt durch den hier bestimmenden ohmschen Widerstand der oben beschriebene lineare Bereich.

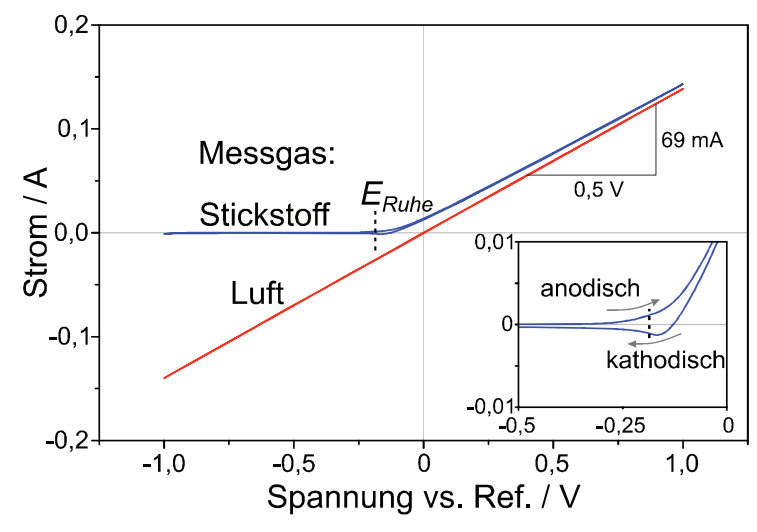

Abb. 2. I-U-Kennlinien des Festelektrolytsensors; Zelltemperatur: $750^{\circ} \mathrm{C}$; Potentialvorschubgeschwindigkeit: $100 \mathrm{mV} \mathrm{s}^{-1}$; Messgasvolumenstrom:

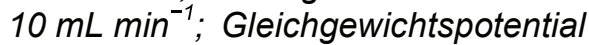
der Kurve in $\mathrm{N}_{2}$ : $-183,5 \mathrm{mV}$ (gestrichelte Linien); Inset: Vergrößerung der Kurve mit $\mathrm{N}_{2}$ als Messgas im Bereich von $-0,5$ bis $0 \mathrm{~V}$.

In kathodische Richtung befindet sich in der Nähe des Gleichgewichtspotentials ein Peak (siehe Inset in Abb. 2). Es wird davon ausgegangen, dass dieser hauptsächlich auftritt, weil Sauerstoff, der zuvor bei positiven Spannungen in das Zellinnere gepumpt wurde, noch nicht vollständig mit dem Messgasstrom aus der Zelle ausgespült wurde. In diesem Fall entsteht der $\mathrm{O}_{2}$-Reduktionspeak bei dieser Zelle nicht wie sonst in der Voltammetrie durch Diffusionslimitierungen, sondern hauptsächlich durch Ausspülen von $\mathrm{O}_{2}$ aus dem Zellinneren. Weiterhin ist in Abb. 2 zum Vergleich die I-UKennlinie einer gassymmetrischen FestelektroIytzelle, bei der an beiden Elektroden Luft anliegt, dargestellt (rote Gerade). Die hohe Sauerstoffverfügbarkeit im Messgas Luft bewirkt, dass auch bei hohen Überspannungen keine Diffusionslimitierung zu beobachten ist. Für den untersuchten Spannungsbereich ergibt sich folglich ein linearer Stromverlauf. Durch die Gassymmetrie verläuft das Stromsignal punktsymmetrisch um den Koordinatenursprung, und das Gleichgewichtspotential der Messgaselektrode beträgt unter Vernachlässigung thermisch bedingter Asymmetriespannungen genau $0 \mathrm{~V}$ gegenüber der Referenzelektrode. 
Der aus dem Anstieg des linearen Stromverlaufs berechnete Gesamtwiderstand der Zelle beträgt $R_{\text {ges }}=7,2 \Omega$. Er repräsentiert die Summe aus den Polarisationswiderständen $R_{p o l}$ beider Elektroden und dem Elektrolytwiderstand $R_{\mathrm{el}}$. Letzterer lässt sich aus der ionischen Leitfähigkeit (nach [3]: $2,07 \mathrm{~S} \mathrm{~m}^{-1}$ ) sowie den Abmessungen des Festelektrolyts $(A=$ $\left.8,72 \mathrm{~cm}^{2}, d=1,0 \mathrm{~mm}\right)$ zu $0,55 \Omega$ berechnen. Der mittlere Polarisationswiderstand der Elektroden beträgt demnach etwa $30 \Omega \mathrm{cm}^{2}$.

Für weitere Untersuchungen an Zellen mit getrennten Gasräumen ist der negative Spannungsbereich von Interesse, in dem die Oxidations- und Reduktionsprozesse der hier interessierenden Analyte Wasserstoff und Sauerstoff an der Messgaselektrode dominieren.

\section{Peakausbildung an $\mathrm{H}_{2}$ - und $\mathrm{O}_{2}$-haltigen Messgasen}

Mittels der Cyclovoltammetrie wurden Versuchsbedingungen gesucht, bei denen es für stickstoffhaltige Messgase, die Spuren von $\mathrm{H}_{2}$, $\mathrm{O}_{2}$ oder $\mathrm{H}_{2} \mathrm{O}$ enthalten, zu den bereits bekannten Zusammenhängen der Voltammetrie bezüglich der Peakhöhe kommt. Dazu wurden die Zelltemperatur, die Elektrodenfläche sowie die Potentialvorschubgeschwindigkeit variiert. Die Ergebnisse der Untersuchungen werden im Folgenden erläutert.

Die bei unterschiedlichen Zelltemperaturen aufgenommenen Cyclovoltammogramme $\mathrm{O}_{2-}$ haltiger Messgase sind in Abb. 3 abgebildet. Erwartungsgemäß wird der in kathodische Richtung auftretende Sauerstoff-Reduktionspeak mit steigender Temperatur größer und das Maximum verschiebt sich zu positiveren Spannungen. Der Peak erreicht bei Temperaturen oberhalb von $600^{\circ} \mathrm{C}$ eine Fläche, die eine quantitative Auswertung ermöglicht.

Die in Abb. 4 dargestellten Voltammogramme bei unterschiedlichen Elektrodengrößen belegen, dass sich die Elektrodenfläche auf die Hysterese des Stromes zwischen kathodischer und anodischer Richtung auswirkt. Je größer die Elektrode, desto größer ist der kapazitive Strom, der für die Umladung der Elektroden und Ausbildung von elektrochemischen Doppelschichten sorgt. Für cyclovoltammetrische Untersuchungen sind deshalb möglichst kleine Elektroden zu nutzen, um solche bei analytischen Applikationen unerwünschten kapazitiven Ströme gering zu halten.

Bei der klassischen Voltammetrie an Zellen mit wässrigem Elektrolyt steigt die Höhe eines Peaks im Voltammogramm gemäß der Randles-Sevčik-Gleichung proportional zur Wurzel der Potentialvorschubgeschwindigkeit $\mathrm{dU}_{\mathrm{p}} / \mathrm{dt}$. Es wurde untersucht, ob sich dieser Zusammenhang an Festelektrolytzellen auf gleiche Weise einstellt. Abb. 5 zeigt Cyclovoltammogramme, die bei unterschiedlichen Potential- vorschubgeschwindigkeiten an einer Zelle des Typs „ „H $\mathrm{H}_{2}, \mathrm{H}_{2} \mathrm{O}, \mathrm{N}_{2}, \mathrm{Pt}$ YYSZ| Pt, Luft" aufgenommen wurden.

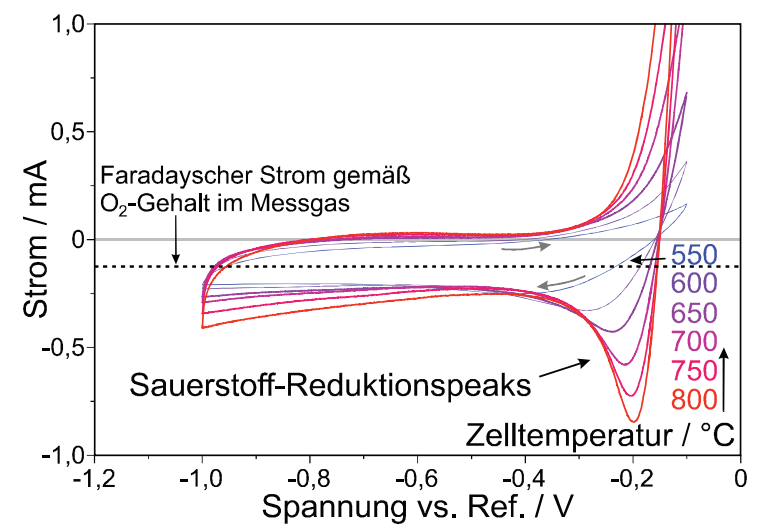

Abb. 3. Cyclovoltammogramme zur Variation der Zelltemperatur; Messgas: 40 Vol.-ppm $\mathrm{O}_{2}$ in $\mathrm{N}_{2}$; Messgasvolumenstrom: $10 \mathrm{~mL} \mathrm{~min}^{-1}$; Elektrodenfläche: $\quad 0,75 \mathrm{~cm}^{2}$; Ruhepotential: $-150 \mathrm{mV} \quad\left(550^{\circ} \mathrm{C}\right)$ bis $-200 \mathrm{mV}$ $\left(800^{\circ} \mathrm{C}\right)$; Potentialvorschubgeschw.: $1 \mathrm{~V} \mathrm{~s}^{-1}$; Startpotential: $-0,17 \mathrm{~V}$; dargestellt ist jeweils der zweite Zyklus.

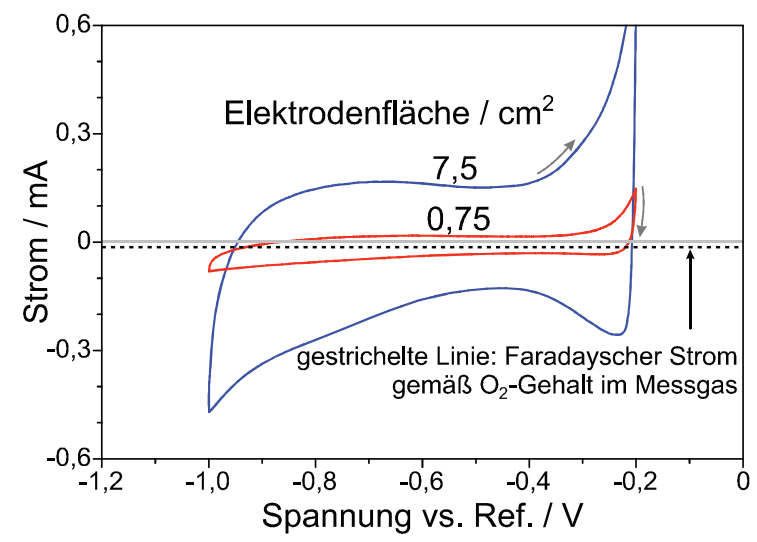

Abb. 4. Cyclovoltammogramme zur Variation der Elektrodengröße; Messgas: $5 \mathrm{Vol}$.-ppm $\mathrm{O}_{2}$ in $\mathrm{N}_{2}$; Messgasvolumenstrom: $10 \mathrm{~mL} \mathrm{~min}^{-1}$; Zelltemperatur: $750{ }^{\circ} \mathrm{C}$; Ruhepotential: $-235 \mathrm{mV}$; Potentialvorschubgeschw.:

$200 \mathrm{mV} \mathrm{s}^{-1}$; Startpotential: $-0,20 \mathrm{~V}$; dargestellt ist jeweils der zweite Zyklus.

Obwohl im gesamten untersuchten Potentialbereich ein nahezu vollständiger Umsatz des Wasserstoffs gemäß dem faradayschen Gesetz auftritt, kommt es zur zusätzlichen Ausbildung von $\mathrm{H}_{2}$-Oxidationspeaks im Bereich zwischen $-0,35$ und $-0,2 \mathrm{~V}$. Diese Peaks treten als Schulter im exponentiell anwachsenden $\mathrm{O}_{2}$-Pumpstrom (in Abb. 5 gestrichelt dargestellt) auf. Zur Auswertung der Peakhöhen wurde jeweils der $\mathrm{O}_{2}$-Pumpstrom als Basislinie vom Messsignal subtrahiert. 


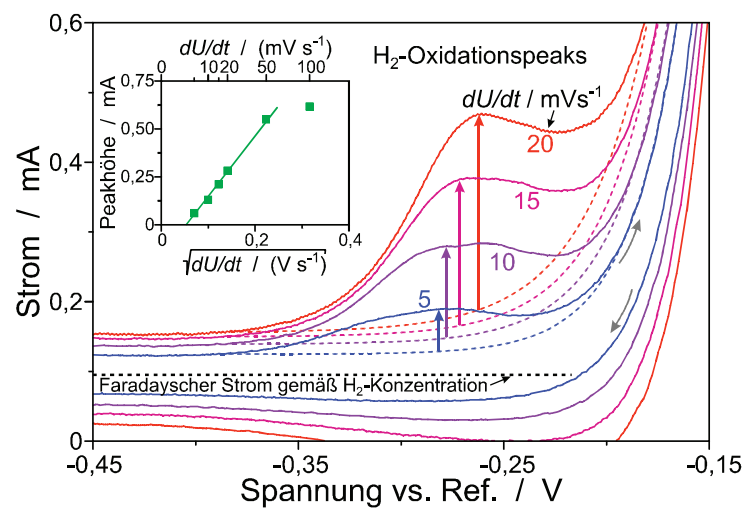

Abb. 5. Cyclovoltammogramme zur Variation der Potentialvorschubgeschwindigkeit; die Basislinien (gestrichelt, farbig) sind die exponentiellen Stromanstiege, die den bei wachsenden Spannungen zunehmenden Sauerstoff-Pumpströmen entsprechen; die Pfeillängen entsprechen den jeweiligen Höhen der $\mathrm{H}_{2}$-Oxidationspeaks; Inset: Peakhöhen der $\mathrm{H}_{2}$-Oxidationspeaks in Abhängigkeit der Wurzel der Potentialvorschubgeschw.; Elektrodenfläche: $7,5 \mathrm{~cm}^{2}$; Zelltemperatur: $800{ }^{\circ} \mathrm{C}$; Messgas: $66 \mathrm{Vol}$.-ppm $\mathrm{H}_{2}+$ 34 Vol.-ppm $\mathrm{H}_{2} \mathrm{O}$ in $\mathrm{N}_{2}$; Messgasvolumenstrom: $10 \mathrm{~mL} \mathrm{~min}^{-1}$; Ruhepotential: $-971,1 \mathrm{mV}$; dargestellt ist jeweils der zweite Zyklus.

Wie im Inset von Abb. 5 dargestellt ist, nimmt bis $\mathrm{zu} \mathrm{dU}_{\mathrm{p}} / \mathrm{dt}=50 \mathrm{mV} \mathrm{s}^{-1}$ die Peakhöhe linear mit der Wurzel der Potentialvorschubgeschwindigkeit zu. Bei Potentialvorschubgeschwindigkeiten größer $50 \mathrm{mV} \mathrm{s}^{-1}$ gehen die $\mathrm{H}_{2}$-Oxidationspeaks in den exponentiellen Stromanstieg der $\mathrm{O}_{2}$-Bildung über, was mit einer geringeren Zunahme der auswertbaren Peakfläche verbunden ist und in einem Knick in der Auftragung der Peakhöhe in Abhängigkeit von der Wurzel der Potentialvorschubgeschwindigkeit resultiert.

Aus den erzielten Messdaten zur Variation von $\mathrm{dU}_{\mathrm{p}} / \mathrm{dt}$ lässt sich schlussfolgern, dass die klassischen Zusammenhänge der Voltammetrie auch bei Festelektrolytzellen gelten, sogar für vergleichsweise große Elektroden.

\section{Sensitivität des Sensors auf $\mathrm{H}_{2}$ bzw. $\mathrm{O}_{2}$}

Die Sensitivität des cyclovoltammetrisch betriebenen Festelektrolytsensors wurde für $\mathrm{H}_{2}$ und $\mathrm{O}_{2}$ unter Anwendung der gefundenen optimierten Versuchsbedingungen untersucht.

Ergebnisse für Messgase unterschiedlicher $\mathrm{H}_{2}-$ Konzentration sind in Abb. 6 dargestellt. Die Peakauswertung erfolgte wie oben beschrieben durch Subtraktion der exponentiell ansteigenden Basislinie. Die in Abb. 7 gezeigten resultierenden Peakdaten zeigen, dass Peakhöhe und -fläche bis zu einer $\mathrm{H}_{2-}$
Konzentration von etwa 100 Vol.-ppm linear wachsen und oberhalb dieser Konzentration nicht mehr wesentlich größer werden, da der Peak zunehmend in den exponentiellen Stromanstieg der $\mathrm{O}_{2}$-Bildung übergeht.

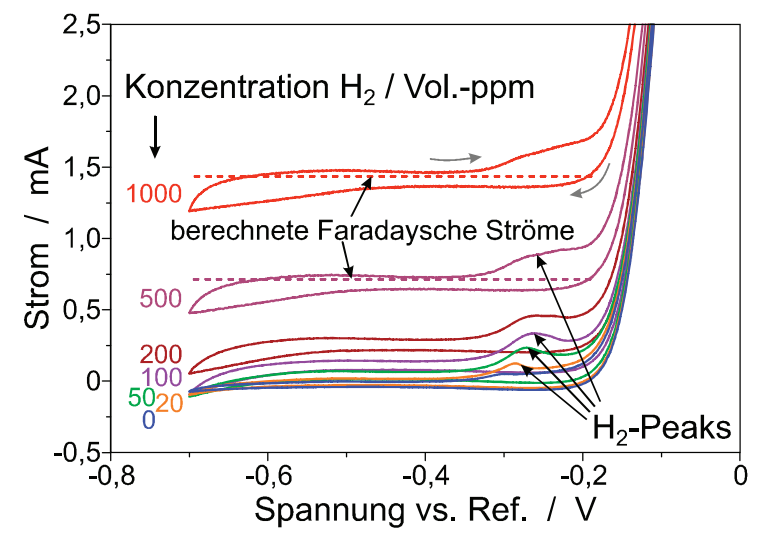

Abb. 6. Cyclovoltammogramme an Messgasen unterschiedlicher $\mathrm{H}_{2}$-Konzentrationen; Zelltemperatur: $750^{\circ} \mathrm{C}$, Messgasvolumenstrom: $10 \mathrm{~mL} \mathrm{~min}^{-1}$, Elektrodenfläche: $7,5 \mathrm{~cm}^{2}$, Potentialvorschubgeschwindigkeit: $10 \mathrm{mV} \mathrm{s}^{-1}$.

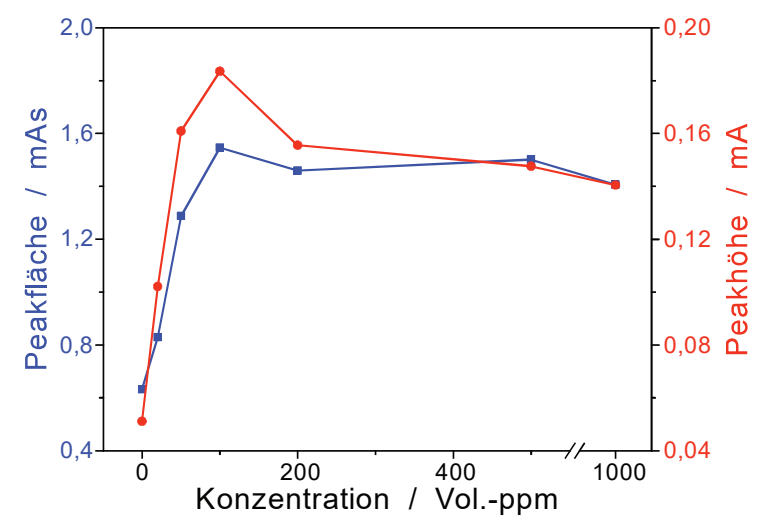

Abb. 7. Höhen und Flächen der in Abb. 6 gezeigten $\mathrm{H}_{2}$-Peaks in Abhängigkeit von der Konzentration.

Bei den Voltammogrammen fällt auf, dass eine Änderung der Konzentration eine Parallelverschiebung der Kurven in Richtung der Stromachse bewirkt: je höher die Konzentration, desto größere Ströme fließen im gesamten untersuchten Potentialbereich. Hier wird der dynamische, potentialabhängige Strom von einem faradayschen, potentialunabhängigen Stromfluss überlagert. Für 500 und 1000 Vol.ppm sind die berechneten faradayschen Ströme eingezeichnet, sie liegen genau im Strombereich der jeweiligen gemessenen Voltammogramme. Möglich wird dieser faradaysche Umsatz durch die vergleichsweise großen Elektrodenflächen, durch den kontinuierlichen Zufluss von mit $\mathrm{H}_{2}$ beladenem Messgas und dadurch, dass im hier gezeigten Potentialbereich $\mathrm{H}_{2}$ immer zu einem wesentlichen Anteil oxidiert wird. 
An Messgasen mit unterschiedlichen $\mathrm{O}_{2}$ Konzentrationen wurden die in Abb. 8 gezeigten Cyclovoltammogramme aufgenommen. Daraus wird ersichtlich, dass erwartungsgemäß auch bei $\mathrm{O}_{2}$ eine Konzentrationsänderung eine Verschiebung der Messkurven entlang der Stromachse bewirkt.

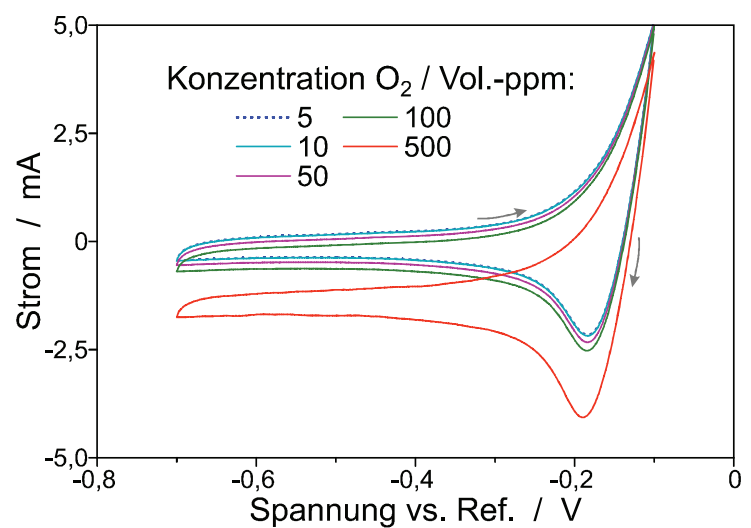

Abb. 8. Cyclovoltammogramme an Messgasen unterschiedlicher SauerstoffKonzentrationen; Potentialvorschubgeschw.: $200 \mathrm{mV} \mathrm{s}^{-1}$; Elektrodenfläche: 7,5 $\mathrm{cm}^{2}$; Ruhepot.: $-241 \mathrm{mV}$ $\left(5 \mathrm{Vol}-\mathrm{ppm} \quad \mathrm{O}_{2}\right.$ ) bis $-135 \mathrm{mV}$ (500 Vol.-ppm $\quad \mathrm{O}_{2}$ ); Messgasvolumenstrom: $10 \mathrm{~mL} \mathrm{~min}^{-1}$; Zelltemperatur: $750{ }^{\circ} \mathrm{C}$; dargestellt ist jeweils der 2. Zyklus.

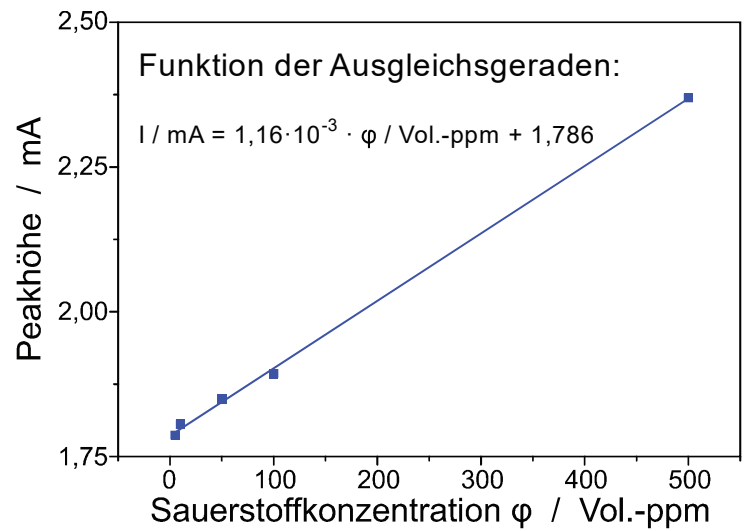

Abb. 9. Höhen der $\mathrm{O}_{2}$-Peaks in den Cyclovoltammogrammen von Abb. 8; die Peakhöhen wurden durch Differenzbildung zwischen den jeweiligen Absolutströmen am Peakminimum und bei $-0,55 \mathrm{~V}$ ermittelt.

Aus den Kalibrierdaten, die in Abb. 9 gezeigt sind, geht eine lineare Änderung der Höhe des $\mathrm{O}_{2}$-Reduktionspeaks mit der Konzentration hervor. Die Ausgleichsgerade dieser Kalibrierung verläuft nicht durch den Koordinatenursprung, da während jedes Messzyklus zusätzlicher Sauerstoff ins Messgas gepumpt wird (bei Potentialen oberhalb von etwa $-0,15 \mathrm{~V}$; siehe Abschnitt „Peakausbildung bei redoxaktiven Substanzen"). Die Kalibrierergebnisse belegen die zuverlässige Bestimmbarkeit relativ kleiner $\mathrm{O}_{2}$-Konzentrationen im Messgas trotz der deutlich höheren $\mathrm{O}_{2}$-Mengen, die während der Messung ins Messgas gepumpt werden.

\section{Querempfindlichkeiten}

Abb. 10 zeigt Voltammogramme von Gasgemischen, die 200 Vol.-ppm $\mathrm{H}_{2}$ in $\mathrm{N}_{2}$ enthalten und die bei unterschiedlichen Messgasvolumenströmen im Bereich von 5 bis $50 \mathrm{~mL} / \mathrm{min}$ jeweils aufgenommen wurden.

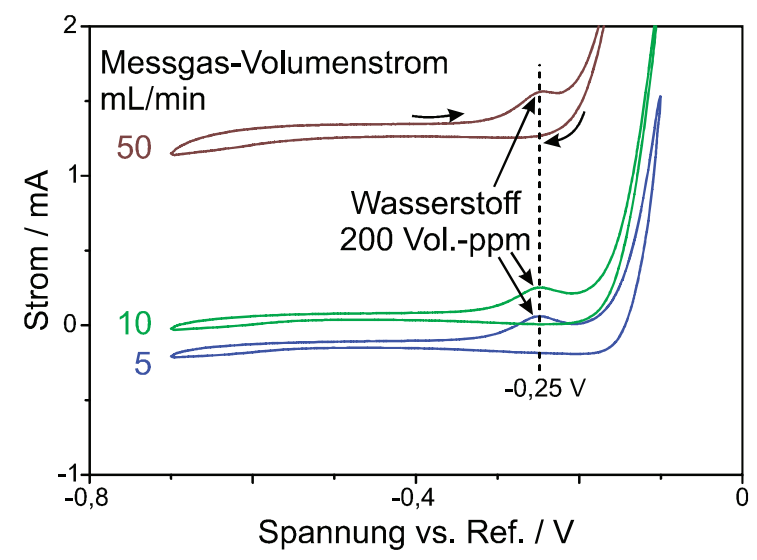

Abb. 10. Cyclovoltammogramme an Messgasen mit jeweils 200 Vol.-ppm $\mathrm{H}_{2}$ bei unterschiedlichen Volumenströmen; Zelltemperatur: $700{ }^{\circ} \mathrm{C}$; Potentialvorschubgeschw.: $10 \mathrm{mV} / \mathrm{s}$; Messgasvolumenstr.: 5 bis $50 \mathrm{~mL} / \mathrm{min}$; Elektrodenfläche: $7,5 \mathrm{~cm}^{2}$; Ruhepotentiale: $-161 \mathrm{mV}$ (5 mL/min) bis $-1030 \mathrm{mV}$ (50 mL/min); dargestellt ist jeweils der zweite Zyklus.

Die in Tab. 1 aufgeführten Daten belegen, dass sowohl die Lage als auch die Höhe der $\mathrm{H}_{2}$-Oxidationspeaks unabhängig vom Volumenstrom sind.

Tab. 1: Peakdaten der $\mathrm{H}_{2}$-Oxidationspeaks aus Abb. 10.

\begin{tabular}{|c|c|c|}
\hline $\begin{array}{c}\text { Messgasvol.- } \\
\text { str. / mL/min }\end{array}$ & $\begin{array}{c}\text { Peakzentrum / } \\
\text { V }\end{array}$ & $\begin{array}{c}\text { Peakhöhe / } \\
\mathrm{mA}\end{array}$ \\
\hline 5 & $-0,250$ & 0,149 \\
\hline 10 & $-0,250$ & 0,153 \\
\hline 50 & $-0,250$ & 0,146 \\
\hline
\end{tabular}

Bei der Aufnahme der in Abb. 10 gezeigten Cyclovoltammogramme kam es durch geringfügige Leckagen zu einem Sauerstoffeinbruch ins Messgas. Durch die Variation des Volumenstroms resultierten bei konstanter Leckage unterschiedliche Sauerstoffkonzentrationen im Messgas. Diese wurden anhand der Ruhespannungen berechnet (jeweils unmittelbar vor den Cyclovoltammogrammen aufgezeichnet) und sind in Tab. 2 aufgeführt. 
Tab. 2: Sauerstoffkonzentrationen in den Messgasen der in Abb. 10 gezeigten Cyclovoltammogramme.

\begin{tabular}{|c|c|c|}
\hline $\begin{array}{c}\text { Messgasvol.- } \\
\text { str. / mL/min }\end{array}$ & $\begin{array}{c}\text { Ruhespan- } \\
\text { nung / mV }\end{array}$ & $\begin{array}{c}\mathrm{O}_{2} \text {-Konz. I } \\
\text { Vol.-ppm }\end{array}$ \\
\hline 5 & -161 & 20 \\
\hline 10 & -770 & 100 \\
\hline 50 & -1030 & 195 \\
\hline
\end{tabular}

Der unterschiedliche Sauerstoffgehalt wirkt sich hier zwar auf die Höhe des Grundstromes, nicht aber auf die Höhe der interessierenden $\mathrm{H}_{2}$-Oxidationspeaks und deren Potential aus, das jeweils bei ca. $-0,25 \mathrm{~V}$ liegt. Ursache für den mit steigender $\mathrm{O}_{2}$-Konzentration abnehmenden Grundstrom ist das wachsende Verhältnis von reduzierbaren zu oxidierbaren Bestandteilen, die pro Zeiteinheit an die Messgaselektrode gelangen.

\section{Schlussfolgerungen}

An einem cyclovoltammetrisch betriebenen kommerziell verfügbaren Festelektrolytsensor konnten im Durchflussmodus Betriebsparameter gefunden werden, bei denen es für $\mathrm{H}_{2}$ - und $\mathrm{O}_{2}$-haltige Gasgemische zur Ausbildung charakteristischer Peaks kommt.

Im unteren Konzentrationsbereich gibt es für jedes dieser Gase einen linearen Zusammenhang zwischen der Konzentration und den auswertbaren Peakeigenschaften Höhe und Fläche, für $\mathrm{H}_{2}$ : bis $100 \mathrm{Vol}$-ppm, für $\mathrm{O}_{2} \mathrm{im}$ gesamten untersuchten Konzentrationsbereich bis 500 Vol.-ppm.

Während sich die $\mathrm{H}_{2}$-Oxidationspeaks in Cyclovoltammogrammen ausbilden, die bei Potentialvorschubgeschwindigkeiten bis $100 \mathrm{mV} / \mathrm{s}$ aufgezeichnet werden, treten die $\mathrm{O}_{2}$-Reduktionspeaks bei Potentialvorschubgeschwindigkeiten im Bereich oberhalb von 200 bis über $1000 \mathrm{mV} / \mathrm{s}$ auf.

Auf diese Weise konnte gezeigt werden, dass mit dem hier beschriebenen Sensor Wasserstoff in einem $\mathrm{H}_{2} / \mathrm{O}_{2}$-Gasgemisch mit hoher Selektivität bestimmt werden kann.

\section{Danksagung}

Die Untersuchungen wurden teilweise im Rahmen der mit Mitteln der Bundesministerien für Umwelt, Naturschutz und Reaktorsicherheit (BMU) sowie für Ernährung, Landwirtschaft und Verbraucherschutz (BMELV) geförderten Forschungsprojekte VESBA (FK 03KB045A) bzw. LIMOS (FK 22011110) durchgeführt. Die Verantwortung für den Inhalt dieser Arbeit liegt bei den Autoren, die für die Förderung danken.

\section{Literaturnachweis}

[1] G. Henze in „Polarographie und Voltammetrie: Grundlagen und analytische Praxis", Springer, Berlin, 22-65 (2001), Kap. Methoden.

[2] M. Schelter, J. Zosel, W. Oelßner, U. Guth, M. Mertig, Sensors and Actuators B: Chemical 187, 209-214 (2012); doi: 10.1016/j.snb.2012.10.111.

[3] J.-H. Park, R. N. Blumenthal, Journal of the Electrochemical Society 136, 2867-2876 (1989); doi: 10.1149/1.2096302. 